\title{
Cheap talk and reputation in repeated pre-trial negotiation
}

\author{
Jeong-Yoo Kim*
}

December 1, 1993

\begin{abstract}
Infinitely repeated interaction between a defendant and a plaintiff can enhance the credibility of cheap talk and improve efficiency in outcome that would be infeasible without cheap talk. The basic driving force is reputation effect. If the players are concerned about their reputation, cheap talk cannot be taken as meaningless even in a game where the interests of the players are sufficiently conflicting, because possible current gains from opportunistic behavior can be wiped out by future losses in payoff from damaged reputation.
\end{abstract}

\section{Introduction}

"A certain shepherd's boy kept his sheep upon a common, and in sport and wantonness would often cry out, the wolf, the wolf. By this means, he several times drew the husbandmen in an adjoining field, from their work; who finding themselves deluded, resolved for the future to take no notice of his alarm. Soon after the wolf came indeed, the boy cried out in earnest. But no heed being given to his cries, the sheep are devoured by the wolf." The Shepherd's Boy and The Wolf, Aesop's Fables

\footnotetext{
${ }^{*}$ Hong Ik University
}

I am greatly indebted to my advisor, Jeroen Swinkels for his constant encouragement. I also would like to thank Vincent Crawford, Joseph Farrell and Joel Sobel for their helpful comments, and Mitchell Polinsky, Bernard Salanie, Ennio Stacchetti, David Starrett and Robert Wilson for helpful conversation and advice. The financial support from Stanford Center on Conflict and Negotiation is gratefully acknowledged. 
Parties involved in a legal dispute often communicate with each other before or during a settlement negotiation. Nobody would believe that such communication can have no influence on the outcome of the negotiation at all. Nevertheless, very little has been said in the literature about the role of communication ${ }^{1}$ in pre-trial bargaining.

Since Crawford and Sobel (1982) showed that cheap talk ${ }^{2}$ may not be so "cheap" if the parties have common interest in some degree, many authors have analyzed the role of cheap talk in a specific setting; for instance, in a double auction (Farrell and Gibbons, 1989; Matthews and Postlewaite, 1989), in the decision of entry into natural monopoly industry (Farrell, 1987), in adopting a new technology (Farrell and Saloner, 1985), in the budgetary process (Matthews, 1989). Recently, Kim (1993) allowed for the possibility of pre-play communication in pre-trial negotiation, showing that if bargaining costs are reasonably high, costless communication (cheap talk) followed by serious pre-trial negotiation may help legal parties to avoid costly litigation by effectively signalling private information of the informed party which it is mutually beneficial to know.

In this paper, I study the role of cheap talk in the repeated relationship between a defendant and a plaintiff. The main result is that repeated interaction between a defendant and a plaintiff can make cheap talk convey some useful information even if the bargaining costs are negligible. The intuition is that if the players are concerned about their reputation, cheap talk cannot be taken as meaningless even in a game where the interests of the players are so conflicting that cheap talk would be meaningless if they met only once, because possible current gains from opportunistic behavior can be wiped out by future losses in payoff from damaged reputation.

Consider the relationship between a firm and a labor union as an example. The workers are exposed to some risk that might be attributable to the carelessness of the firm at all times. If an accident occurs, the labor union may sue against the firm ${ }^{3}$ or on the threat of doing it, may extract the settlement amount, and this defendant-plaintiff relationship between the firm and the labor union will be repeated over and over again. Unlike in a one-shot game, if the relationship is rather perpetual, the union will find it to its advantage to maintain some level of credibility in cheap talk because of the reputation effect. That is, the reputation can discipline the plaintiff's cheap talk language in some degree.

This long-term relationship between a plaintiff and a defendant can also be found in repeated business transactions, such as franchise contracts in retail businesses or contracts between a large manufacturer and a small company that supplies the manufacturer with one of the components. If the purchaser found a defect in the goods after a purchase, he would 
inform the supplier of his losses for a compensation on an informal basis rather than prepare the accurate estimate for the losses with considerable costs or directly appeal to legal rights in a court of law.

There are other examples for this relationship abundant in business or personal life, such as movie distributor vs. censorship board, developer vs. suburban municipality, regulatory agency vs. firms of regulated industry etc.. If the relationship is expected to endure over a long period of time in this way, the informal understandings become more important than formal agreements. Instead of engaging in sharp dealing, the parties are likely to rely upon informal devices to control their relationship. Thus, if one party comes to regard the other as taking too much advantage of this informal mechanism or even cheating in the relationship, the deceptive party may be brought back into line by a response in-kind.

For the purpose of incorporating this idea, the model to be adapted in this paper will be basically a repeated game with cheap talk. In this setup of the repeated game, the communicative equilibrium that is implied by the main theorem takes the following form; each player chooses a randomized strategy i.e., the plaintiff who will be assumed to be the informed party exaggerates his damage amount with strictly positive probability and the defendant who will be assumed to be the uninformed party checks up on honesty of the plaintiff by taking the action which is not in his one-time interest with strictly positive probability to figure out whether the opponent made an honest report or not. The outcome of this repeated litigation game with cheap talk may seem similar to the one of the earlier model (Kim, 1993) in the sense that cheap talk could help coordination between the plaintiff and the defendant in both models, but the mechanisms through which cheap talk contributes to coordination are quite different in two models. In the former, it is the defendant's monitoring behavior (the possibility of trial) that differentiates the signalling costs of the possible types, whereas, in the latter, it is the significance of the negotiation cost.

The equilibrium outcome is reminiscent of the results of Green and Porter (1986). In both of the models, there is an inherent informational problem: a moral hazard problem in Green and Porter that players cannot observe the opponent's action, and an adverse selection problem in our model that one player cannot observe the other player's actual type. But, in Green and Porter, a commonly observable signal is available that can be used to induce a collusive behavior. Contrary to the Green and Porter's model, this model has no such signal to be used for a trigger strategy. This feature does away with pure strategy equilibria in this model. However, the two models have in common that due to imperfect information, the fully cooperative outcome cannot be supported. It could be supported only if the players 
kept playing cooperatively even after observing an unfavorable signal. But, that would surely yield an incentive to chisel. So, some degree of efficiency loss is indispensable to give the right incentive to cooperate under imperfect information i.e., there is a tradeoff between efficiency and incentive. Thus, full cooperation is inconsistent with imperfect information.

The model in this paper closely follows Kim (1993). The important structural elements of the model are that both litigants are risk neutral, that the plaintiff has information that is not available to the defendant, but the defendant has no information to which the plaintiff does not have access, that the defendant makes a take-it-or-leave-it settlement offer and that the amounts of the settlement offered are exogenous. The only crucial difference is that in this model no legal costs are incurred if the action is filed but settled.

The organization of this paper goes as follows; Section 2 presents a simple model of the litigation game with cheap talk and shows that if the payoffs are conflicting enough, cheap talk will not convey any valuable information in any arbitrary finitely repeated game. In section 3, infinitely repeated cheap talk game will be analyzed and the main theorem will be proved that if the cheap talk game is repeated infinitely many times, more efficient outcome where cheap talk is taken as serious is supported as an equilibrium. In section 4, public randomization will be allowed to show that the players can approximate the most efficient outcome of the one-period game as close as they want by taking the probability of checking which is arbitrarily small but still positive. A brief review of some related theoretical literature is given in section 5 and concluding remarks will follow in Section 6 .

\section{Basic model}

There are one potential defendant $(D)$ and one potential plaintiff $(P)$, both of whom are risk-neutral. Suppose the accident happened and $P$ filed a suit against $D^{4}$. We assume that $P$ knows the actual damage amount $w$ that $D$ does not know, but only knows the probability distribution of. Also, we assume that $q, P^{\prime} s$ winning probability under the given liability rule, is common knowledge. For simplicity, we assume that $w$ is either $w_{l}$ or $w_{h}$ and that $\pi$, the

probability that $w=w_{h}$, is common knowledge. We will denote the plaintiff with private information $w_{h}\left(w_{l}\right.$, resp.) by $P_{h}\left(P_{l}\right.$, resp.). After $P$ filed a suit, informal conversation between $P$ and $D$ takes place. During conversation, various sorts of information can be exchanged. $P$ may inflate the level of damage inflicted or threaten to litigate immediately. Here, we only consider the simplest possible language ${ }^{5}$. Following informal conversation, serious settlement negotiations are conducted. The negotiation procedure is assumed to 
take the following form. First, $D$ chooses a settlement amount and offers it to $P$ on a take-it-or-leave-it basis. $P$ then decides whether to accept the offer. If $P$ has rejected the $D^{\prime} s$ offer, the dispute is settled by the court. The court may be subject to error but we assume that the court has the ability to verify the exact damage amount $w$. We also assume that the American rule, under which each party bears its own legal costs, is prevailing. The litigation costs to the plaintiff and the defendant will be denoted by $c_{p}$ and $c_{d}$, respectively. For simplicity, we make an additional assumption that $D^{\prime} s$ possible settlement offers are exogenously given; it can be either $s_{l}$ or $s_{h}$ satisfying,

$$
\begin{aligned}
& \quad x_{l}-c_{p}<s_{l}<x_{h}-c_{p}<s_{h}<x_{h}+c_{d}{ }^{6} \\
& \text { where } x_{l} \equiv q \times w_{l} \text { and } x_{h} \equiv q \times w_{h} .
\end{aligned}
$$

This assumption guarantees (i) that $s_{l}$ is accepted by $P_{l}$ only, while $s_{h}$ is accepted by both $P_{h}$ and $P_{l}$ and (ii) that $D$ prefers offering $s_{h}$ to $P_{h}$ to being litigated by him.

We will start our analysis with some notation. Let $\Omega$ be the set of the states of the world, $M$ be the set of $P^{\prime} s$ possible messages and $S$ be the set of $D^{\prime} s$ possible actions, where $\Omega \equiv\left\{w_{l}, w_{h}\right\}, M \equiv\{" L ", " H "\}, S \equiv\left\{s_{l}, s_{h}\right\}$. A strategy for $P$ is a function defined on the set of the states of the world:

$$
\sigma^{P}: \Omega \rightarrow \Delta(M)
$$

where $\Delta(M)$ is the set of all probability distributions over $M$.

Similarly, a strategy for $D, \sigma^{D}$, is a function defined on the set of messages by $P$ :

$$
\sigma^{D}: M \rightarrow \Delta(S)
$$

A strategy for $P$ specifies the probability that $P$ announces " $\mathrm{H}$ " for each possible level of damages whereas a strategy for $D$ specifies the probability that $D$ makes a low offer $s_{l}$ for each message. The payoff functions of $P$ and $D$ are denoted by $u^{P}: S \times \Omega \rightarrow R$ and $u^{D}: S \times \Omega \rightarrow R$, respectively.

The solution concept employed throughout this paper will be Kreps and Wilson's Sequential Equilibrium ${ }^{7}$. Usually, a cheap talk game may involve two kinds of equilibria, one in which talk is taken to be meaningless and the other in which talk is meaningful, so that it does alter the outcome of the game in the absence of talk. The latter will be meant by the "communicative equilibrium" 8 ", whereas the former is by the "uncommunicative equilibrium". 
Let $G$ denote this one-shot cheap talk game. We can easily see that $(G)$ has only one equilibrium outcome: Each type announces anything or randomizes between " $L$ " and " $H$ " and $D$ chooses $s_{l}$ if $\pi \leq \pi^{*}$, chooses $s_{h}$ if $\pi \geq \pi^{*}$, where $\pi^{*}=\frac{s_{h}-s_{l}}{x_{h}+c_{d}-s_{l}}$.

Announcement " $H$ " will never be credible, because $P_{l}$ always has an incentive to mimic $P_{h}$. So, the plaintiff does not gain any strategic advantage from his private information. Any attempt of $P$ to avoid costly trial by signalling his private information is frustrated by the incentive of $P_{l}$ to mimic $P_{h}$. This is quite an inefficient outcome in the following sense; Suppose $\pi \geq \pi^{*}$. $P_{h}$ has no way to convince $D$ that he is $P_{h}$, reaching mutually beneficial outcome $\left(-s_{h}, s_{h}\right)$ by inducing $D$ to play $s_{h}$.

The above result is summarized in the following proposition.

Proposition 1 There is no communicative equilibrium in $(G)$. That is, $(G)$ has a unique equilibrium outcome in which $\sigma^{P}(\omega)=r \in[0,1], \forall \omega \in \Omega$, and $\sigma^{D}(m)=1, \forall m \in M$ if $\pi \leq$ $\pi^{*}$ and $\sigma^{D}(m)=0 \forall m \in M$ if $\pi \geq \pi^{*}$, where $\pi^{*}=\frac{s_{h}-s_{l}}{x_{h}+c_{d}-s_{l}}$.

This result can be extended to the case where the cheap talk game $(G)$ is repeated finitely many times.

Consider the following finitely repeated game. Right before t-period stage game is played, the state of the nature, $\omega_{t}$ is realized, $t=1,2, \cdots, T$; whether $P$ is severely damaged $\left(w_{h}\right)$ or slightly damaged $\left(w_{l}\right)$. $P$ is informed of $\omega_{t}$ at the beginning of each period, but $D$ only knows the distribution of $\omega_{t}$. Plaintiff's types in each period are independent draws from the Bernoulli distribution with $\operatorname{Prob}\left[\omega_{t}=w_{h}\right]=\pi$. In each period, cheap talk precedes the litigation game. $D$ can observe the message $P$ sends (cheap talk message) but cannot observe $P^{\prime} s$ private information. So, $D$ does not always learn $\omega_{t}$ after period $t$. It is possible only after he chooses $s_{l}$, because it will be accepted by $P_{l}$ only.

The conventional argument on the finitely repeated game shows that there are no other equilibria than a T-times repetition of the uncommunicative equilibrium. Because the one-shot game has a unique equilibrium outcome, it is easy to see;

At period T, $D$ will never believe whatever $P$ announces. Given this, $P$ has no incentive to build up his reputation at period $T-1$, and so on. So, $P$ has no incentive to be honest, at every period $t=1,2, \cdots, T$. 


\section{Infinitely repeated cheap talk game}

Suppose a plaintiff and a defendant play $(G)$ infinitely many times. It will be shown that unlike in the finitely repeated game, there are communicative equilibria where honest announcements are often made and taken seriously.

Let $\left(G^{T}\right)$ denote the T-fold repetition of $(G)$. Then, we will begin the analysis of $\left(G^{\infty}\right)$ by defining the strategies by $P$ and $D$. History of length $t$, denoted by $h_{t}$ consists of a series of messages and responses, including period $t$. Let $H_{t}$ be the set of histories of length $t$. Then, by convention, we set $H_{0}=\emptyset$ and $H_{t}=M^{t} \times S^{t}, t=1,2, \ldots$ Now, let us define the strategies of $P$ and $D$. A strategy should describe a feasible action for every possible history. But, since $P$ has private information, his strategy should also depend on his type up to $t$. A strategy for $P, \sigma^{P}=\left\{\sigma_{t}^{p}\right\}_{t=1}^{\infty}$ is a sequence of functions defined on the set of histories and the type:

$$
\sigma_{t}^{p}: H_{t-1} \times \Omega \rightarrow \Delta(M)
$$

Similarly, a strategy for $D, \sigma^{D}=\left\{\sigma_{t}^{d}\right\}_{t=1}^{\infty}$ is also a sequence of functions defined on the set of histories:

$$
\sigma_{t}^{D}: H_{t-1} \times M \rightarrow \Delta(S)
$$

Finally, we introduce a system of beliefs, $\boldsymbol{\pi}$ which, given history $h_{t}$, describes the posterior belief on $\omega_{t}$ assessed by $D$. Formally, $\boldsymbol{\pi}=\left\{\pi_{t}\right\}_{t=0}^{\infty}$, where $\pi_{t}: H_{t-1} \times M \rightarrow[0,1], \forall t=$ $0,1,2, \ldots$

Let $\delta(<1)$ be the common discount factor and $\Sigma$ be the set of strategy profiles i.e., $\Sigma=\Sigma^{P} \times \Sigma^{D}$ where $\Sigma^{i}$ is the set of player i's strategies, $i=P, D$. Let $U^{i}: \Sigma \times \Omega^{\infty} \rightarrow \Re$ define the player i's payoff function;

$$
U^{i}(\sigma, \omega)=\frac{1-\delta}{\delta} \sum_{t=1}^{\infty} \delta^{t} u^{i}\left(\sigma, \omega_{t}\right)^{9}
$$

We will focus on trigger-strategy equilibria which have the following configuration: (i) There are a normal phase and a punishment phase. (ii) The game begins with the normal phase. (iii) Detection of a dishonest announcement triggers a punishment phase. (iv) At the end (if any) of the punishment phase, the players revert to the normal phase.

We will also restrict our attention to stationary Markov strategies ${ }^{10}$. Then, the following observations will be useful in characterizing our communicative equilibrium. Letting $\sigma_{t}^{D}\left(h_{t-1}, m_{t}\right)$ and $\sigma_{t}^{P}\left(h_{t-1}, \omega_{t}\right)$ be the probability that $D$ chooses $s_{l}$ given $h_{t-1}, m_{t}$ and the probability that $P$ announces " $H$ " given $h_{t-1}, \omega_{t}$ respectively, 
Proposition 2 In any communicative equilibrium, $\sigma_{t}^{D}\left(h_{t-1}, m_{t}\right)>0, \forall h_{t-1} \in H_{t-1}, \forall m_{t} \in$ $M_{t}, \forall t$.

Remark. The proof shows that if $D$ does not check up on whether $P^{\prime} s$ announcement is true or false at all, i.e., never makes a low settlement offer, $P$ will always announce " $H$ ", thus violating the definition of the communicative equilibrium.

Proof. See the appendix.

Proposition 2 implies that in any communicative equilibrium, $D$ should offer a low settlement amount $s_{l}$ with strictly positive probability even after the message " $H$ " is observed.

Proposition 3 In any communicative equilibrium, $\sigma_{t}^{P}\left(h_{t-1}, \omega_{t}\right)>0, \forall h_{t-1} \in H_{t-1}, \forall \omega_{t} \in$ $\Omega, \forall t$.

Remark. The proof shows that if $P$ always tells the truth, it will be best for $D$ never to check up on whether $P^{\prime} s$ announcement is true or false, which is contradictory to the proposition 2 .

Proof. See the appendix.

Together with proposition 2, proposition 3 has the implication that even though $(G)$ is infinitely repeated, we cannot reach the most efficient outcome where at each period $P$ makes an honest report and, believing it, $D$ makes the corresponding settlement offer to each type who declares himself, hence no trial observed in equilibrium.

Corollary 1 The following strategies and beliefs cannot constitute an equilibrium;

(i) $\sigma_{t}^{P}\left(h_{t-1}, w_{l}\right)=0, \sigma_{t}^{P}\left(h_{t-1}, w_{h}\right)=1, \forall h_{t-1} \in H_{t-1}, \forall t$.

(ii) $\pi_{t}\left(h_{t-1}, L\right)=0, \pi_{t}\left(h_{t-1}, H\right)=1, \forall h_{t-1} \in H_{t-1}, \forall t$.

(iii) $\sigma_{t}^{D}\left(h_{t-1}, L\right)=1, \sigma_{t}^{D}\left(h_{t-1}, H\right)=0, \forall h_{t-1} \in H_{t-1}, \forall t$.

From this corollary, we can characterize our communicative equilibrium.

Theorem 1 The following strategies and beliefs constitute a communicative equilibrium;

(i) At each period $t, P$ plays the strategy $\sigma_{t}^{P}\left(h_{t-1}, w_{l}\right)=\alpha, \sigma_{t}^{P}\left(h_{t-1}, w_{h}\right)=1, \forall h_{t-1} \in$ $H_{t-1}, \forall t, 0<\alpha<1$, and D's posterior belief is formed by $\pi_{t}=\frac{\pi}{(1-\pi) \alpha+\pi}$ if $m_{t}=H$ and 
otherwise, $\pi_{t}=0$, and $D$ plays the strategy $\sigma_{t}^{D}\left(h_{t-1}, m_{t}\right)=\beta$, if $m_{t}=H, \forall h_{t-1} \in H_{t-1}, \forall t$, $0<\beta<1$ and $\sigma_{t}^{D}\left(h_{t-1}, m_{t}\right)=1$, if $m_{t}=L$, until $m_{t}$ turns out to be false.

(ii) If it is revealed that $m_{t}$ was false, $P$ and $D$ play $\sigma_{t}^{P}\left(h_{t-1}, \omega_{t}\right)=1$ and $\sigma_{t}^{D}\left(h_{t}\right)=$ $1, \forall h_{t} \in H_{t}, \forall \omega_{t} \in \Omega$ for $N$ periods, and then, switch to the strategy (i) thereafter.

(iii) If $D$ deviates from the strategy (ii), $P$ will play $\sigma_{t}^{P}\left(h_{t-1}, \omega_{t}\right)=1, \forall \omega_{t} \in \Omega, \forall t$.

Remark 1 . The equilibrium induced by the above strategies is characterized by a normal phase (coordinative phase) and a punishment phase (non-coordinative phase). Players begin the game in a normal phase. Each player participates in coordinating with probability $1-\alpha$ (or $1-\beta$ resp.) $-P$ makes an honest announcement with probability $1-\alpha$ when he is slightly damaged and $D$ offers a low settlement amount $s_{l}$ with probability $1-\beta$ when he observes the announcement " $\mathrm{H}$ ". If $P$ is detected inflating his real damage amount, it triggers a punishment phase. They revert to the uncommunicative equilibrium (babbling equilibrium) for $N$ periods, where $D$ disregards whatever $P$ announces, and then restart the normal phase.

Remark 2. In this cheap talk game, a communicative equilibrium can be supported by the uncommunicative equilibrium, as in the infinitely repeated prisoner's dilemma game, a collusive outcome is supported by a Pareto-dominated Nash equilibrium.

To identify the equilibrium values for $\alpha, \beta$ and $N$, we will use the dynamic programming technique. Let us begin our analysis with the following notation.

$V=$ the present discounted value (PDV) of P's expected equilibrium gain before he is informed of $\omega$

$V_{H}=$ the PDV of P's expected equilibrium gain given his type is $w_{h}$

$V_{L}=$ the $\mathrm{PDV}$ of $\mathrm{P}$ 's expected equilibrium gain given his type is $w_{l}$

$V_{L}^{l}=$ the PDV of $P_{l}$ 's expected equilibrium gain when he sent the message $L$

$V_{L}^{h}=$ the PDV of $P_{l}$ 's expected equilibrium gain when he sent the message $H$

$V^{p}=$ the PDV of P's expected equilibrium continuation gain since the punishment phase

Then, we must have

$$
\begin{gathered}
V=\pi V_{H}+(1-\pi) V_{L} \\
V_{H}=(1-\beta)\left\{(1-\delta) s_{h}+\delta V\right\}+\beta\left\{(1-\delta)\left(x_{h}-c_{p}\right)+\delta V\right\} \\
V_{L}=(1-\alpha) V_{L}^{l}+\alpha V_{L}^{h}
\end{gathered}
$$




$$
\begin{gathered}
V_{L}^{l}=(1-\delta) s_{l}+\delta V \\
V_{L}^{h}=(1-\beta)\left\{(1-\delta) s_{h}+\delta V\right\}+\beta\left\{(1-\delta) s_{l}+\delta V^{p}\right\} \\
V^{p}=\left(1-\delta^{N}\right)\left\{\pi\left(x_{h}-c_{p}-s_{l}\right)+s_{l}\right\}+\delta^{N} V
\end{gathered}
$$

Similarly, we can define the following D's expected equilibrium losses.

$W=$ the PDV of D's expected equilibrium loss when no message is available

$W_{H}=$ the PDV of D's expected equilibrium loss after he observed " $H$ "

$W_{L}=$ the PDV of D's expected equilibrium loss after he observed " $L$ "

$W_{H}^{l}=$ the PDV of D's expected equilibrium loss when he plays $s_{l}$ after he observed " $H$ "

$W_{H}^{h}=$ the PDV of D's expected equilibrium loss when he plays $s_{h}$ after he observed " $H$ "

$W^{p}=$ the PDV of D's expected equilibrium continuation loss since the punishment phase

Then, the following conditions must be satisfied. Letting $\hat{\pi}=\pi+(1-\pi) \alpha$,

$$
\begin{gathered}
W=\hat{\pi} W_{H}+(1-\hat{\pi}) W_{L} \\
W_{H}=(1-\beta) W_{H}^{h}+\beta W_{H}^{l} \\
W_{L}=(1-\delta) s_{l}+\delta W \\
W_{H}^{h}=(1-\delta) s_{h}+\delta W \\
W_{H}^{l}=\frac{\pi}{\hat{\pi}}\left\{(1-\delta)\left(x_{h}+c_{d}\right)+\delta W\right\}+\frac{(1-\pi) \alpha}{\hat{\pi}}\left\{(1-\delta) s_{l}+\delta W^{p}\right\} \\
W^{p}=\left(1-\delta^{N}\right)\left\{\pi\left(x_{h}+c_{d}-s_{l}\right)+s_{l}\right\}+\delta^{N} W
\end{gathered}
$$

Also, since $\alpha^{*} \in(0,1), P_{l}$ should be indifferent between being honest and dishonest. So,

$$
V_{L}^{l}=V_{L}^{h}
$$

Similarly, from $\beta^{*} \in(0,1), D$ who has received the message "H" should be indifferent between making a low settlement offer and a high settlement offer. Therefore,

$$
W_{H}^{l}=W_{H}^{h}
$$

Equations (1) - (14) give the equilibrium values $\alpha^{*}, \beta^{*}$ and $V, V_{H}, V_{L}, V_{L}^{l}, V_{L}^{h}, V^{p}$, $W, W_{L}, W_{H}, W_{H}^{l}, W_{H}^{h}$ and $W^{p}$. Computing equilibrium values from the above system of 14 equations appears to be rather complicated, but the following proposition will make the computation straightforward. 
Proposition 4 In a communicative equilibrium $\left(0<\beta^{*}<1\right)$,

(i) $\alpha^{*}=\frac{\left(x_{h}+c_{d}-s_{l}\right) \pi}{\left(s_{h}-s_{l}\right)(1-\pi)} 11$.

(ii) $W=W^{p}=\pi\left(x_{h}+c_{d}-s_{l}\right)+s_{l}$.

Proof. See the appendix.

Surprisingly, $D$ is no better off with cheap talk than without cheap talk! Since $P$ moves first, he can extract the entire efficiency gain partly retrieved by informational transmission, by choosing $\alpha$ strategically so as to induce $D$ to randomize between two choices, $s_{l}$ and $s_{h}$, which leaves $D$ no better than without cheap talk. The share of $D$ in efficiency gain achieved by $D^{\prime} s$ random play of a coordinative action $\left(s_{l}\right)$ is exactly offset by the loss incurred by sometimes being deluded by $P$. That is, cheap talk precedent to each stage game may be useful for $P$ to improve his welfare but not for $D$.

It is also worth noticing that sequential rationality ${ }^{12}$ does not allow much discretion in choosing the length of the punishment periods. For our equilibrium strategies proposed in theorem 1 to be sequentially rational, $N$ should be long enough to punish the deviant, but also not too long for the threat to punish to be credible. Notice that if $\pi<\pi^{*}$, it is an (uncommunicative) equilibrium that $P$ announces " $\mathrm{H}$ " and $D$ chooses $s_{l}$, so that nobody has an incentive to deviate from the strategy (ii) for any $N$. But, if $\pi>\pi^{*}$, to punish the deviant i.e., choose $s_{l}$ hurts $D$ himself. So, it seems that if $N$ is very large, the above strategy will not be sequentially rational. However, the discussion below shows that if $\pi>\pi^{*}$, sequential rationality is not satisfied for any $N$. Checking the condition on $N$ for the above strategies to be sequentially rational given $\pi$ and $\delta$, it is sufficient to check the condition for unimprovability (A one-shot deviation at the next information set is unprofitable.) by the Howard Principle (1960). By the definition of unimprovability, to punish for $N$ periods should give $D$ a lower loss than to deviate the punishment at the next period only. So, when $\pi>\pi^{*}$, it should be that $W^{p}=\left(1-\delta^{N}\right)\left\{\pi\left(x_{h}+c_{d}-s_{l}\right)+s_{l}\right\}+\delta^{N} W \leq s_{h}$. From proposition $4, W^{p}=W=\pi\left(x_{h}+c_{d}-s_{l}\right)+s_{l}>\pi^{*}\left(x_{h}+c_{d}-s_{l}\right)+s_{l}=\left(s_{h}-s_{l}\right)+s_{l}=s_{h}$. So, the above strategy for any $N$ violates sequential rationality if $\pi>\pi^{*}$ i.e., if $\pi>\pi^{*}$, there exists no communicative equilibria.

Several features deserve to be noticed. First, given $\delta$ and $N$, as $\pi$ rises, the punishment will become a heavy burden to $D$ himself. So, $D$ has an incentive to reduce $\beta^{*}$. On the other hand, a rise in $\pi$ will secure the plaintiff the higher expected gain during the punishment period, so that it alleviates the burden of the punishment to the plaintiff for being dishonest. This, together with D's less inclination to check, gives $P$ a stronger incentive to lie. So, $\alpha^{*}$ 
will become larger. Second, as the players are more patient $(\delta \rightarrow 1)$ or $N$ gets larger, the punishment will be harsher, which lowers $\beta^{*}$.

Now, we can ask the following question. If a large $N$ can facilitate coordination among $P$ and $D$ as noticed above, how far can we reach in terms of efficiency? The following theorem tells us that given $\pi$ and $\delta$, there is an upper bound on the efficiency on our equilibria as $N$ gets arbitrarily large.

Theorem 2 Given $\pi\left(<\pi^{*}\right), \delta(<1)$, there exists a positive lower bound $\beta(\delta, \pi)$ for $\beta^{*}(N, \delta, \pi)$ such that for any $N, \beta^{*}(N, \delta, \pi) \geq \underline{\beta}(\delta, \pi)$.

Proof. See the appendix.

As the second step, we will see how much further we can reach in efficiency as $\delta$ goes to 1 given $N \rightarrow \infty$. The following result shows that as the players are more patient, $\beta^{*}$ will approach zero, so will the likelihood of trial $\left(\pi \beta^{*}\right)$, so that we can succeed in asymptotically achieving one sided efficiency ${ }^{13}$ by the permanent punishment scheme.

Theorem 3 Given $N \rightarrow \infty$,

(i) $\lim _{\delta \rightarrow 1} \underline{\beta}(\delta, \pi)=0, \forall \pi<\pi^{*}$.

(ii) $\lim _{\delta \rightarrow 1} V=\pi s_{h}+(1-\pi) s_{l}$.

Proof. See the appendix.

This result is quite striking, considering that $V=W=\pi s_{h}+(1-\pi) s_{l}$, in the first best world where each type is always truthful and $D$ always believes the message he has received. With more and more patience, $P$ can get asymptotically the best he can, leaving $D$ no better than ever. As seen in figure 1, efficiency that should have been sacrificed due to the

informational problem can only partially recovered by cheap talk because $\alpha^{*}=\frac{\left(x_{h}+c_{d}-s_{l}\right) \pi}{\left(s_{h}-s_{l}\right)(1-\pi)} \neq$ 0 while $\beta^{*} \rightarrow 0$, even when the players are very patient.

\section{[ INSERT FIGURE 1 HERE ]}

At this point, it is worth seeing the intuition of how the assumption [A] works to support communicative equilibria. The assumption $x_{h}-c_{p}<s_{h}<x_{h}+c_{d}$ implies that if the sender 
is $P_{h}$, both $P$ and $D$ prefer $s_{h}$. As a result, $D$ has a way to punish $P$ who misrepresents himself (since $x_{h}-c_{p}<s_{h}$ ) and an incentive to believe what $P$ announces up to a certain degree (since $s_{h}<x_{h}+c_{d}$ ). If $s_{h} \leq x_{h}-c_{p}$, there remains an incentive for $P_{l}$ to misrepresent himself, but no way to punish him. Then, even if there is room for improvement in efficiency $\left(c_{p}+c_{d}>0\right)$, communicative equilibria are not viable.

Before closing this section, we will compare the likelihood of trial under two alternative fee systems; the American system whereby each party bears his own litigation costs and the British fee system whereby the loser bears all the litigation $\operatorname{costs}^{14}$.

Corollary 2 Assume that the possible settlement amounts are exogenously given. Then, the likelihood of trial under the British system will be greater than under the American system iff $q>\frac{c_{d}}{c_{p}+c_{d}}$, where $q$ is the probability of $P$ 's winning at trial.

Proof. Let $\beta_{i}^{*}$ be the equilibrium value for $\beta$ under the fee system $i$. From the argument above,

$$
\beta_{i}^{*}=\frac{(1-\delta)\left(s_{h}-s_{l}\right)}{\pi \delta\left(1-\delta^{N}\right)\left(s_{h}-x_{h}+c_{p}^{i}\right)},
$$

where $c_{p}^{i}$ is the litigation costs of the plaintiff under the fee system $i, i=A, B$ and $c_{p}^{A}=$ $c_{p}, c_{p}^{B}=(1-q)\left(c_{p}+c_{d}\right)$. Since the equilibrium likelihood of trial in this model is $\pi \beta_{i}^{*}$, trial is more likely to occur under the British fee system iff $c_{p}^{A}>c_{p}^{B}$ i.e., $q>\frac{c_{d}}{c_{p}+c_{d}}$. Q.E.D.

The intuition behind this corollary is the following; In this model, two separate forces discipline the plaintiff's incentive to lie, the plaintiff's litigation costs and the defendant's checking behavior. If $q>\frac{c_{d}}{c_{p}+c_{d}}$, the costs that the plaintiff has to bear in case that a case is litigated are greater under the American fee system. Since the plaintiff's litigation costs are larger, smaller checking frequency is needed for $D$ to induce $P^{\prime} s$ optimal behavior, which leads to more settlement under the American fee system.

\section{Public randomization ${ }^{15}$}

The arguments so far rely on the implicit assumption that the past mixed strategies are unobservable. In this section, we will allow the possibility of public randomization; put differently, we will assume that $D^{\prime} s$ past mixed strategy can be observable. The idea of public randomization can be justified in many situations. A prominent example is the way a patrol car stops vehicles to detect drunken drivers. It is usual that the police stops one per the fixed number of cars passing by and that the frequency used is observable to the 
frequent drivers. Another example is how the IRS verifies the taxpayer's income. Usually, the taxpayer is able to infer what verification frequency the IRS has actually used from the IRS report or other sources.

Here, we will show that, by allowing public randomization device, the players can achieve asymptotically the full efficiency if $\delta$ is sufficiently large. We will follow the same assumptions and notations as in section 3 except that $D^{\prime} s$ past mixed strategy can be a part of history i.e., $H_{t}=M^{t} \times \Delta(S)^{t}$. Now, it is easy to see that the counterpart of proposition 2 holds.

Proposition 5 Assume that $D^{\prime}$ s mixed strategy is observable. In any communicative equilibrium of $\left(G^{\infty}\right), \sigma_{t}^{D}\left(h_{t-1}, m\right)>0, \forall h_{t-1} \in H_{t-1}, \forall m \in M, \forall t$.

This implies that we cannot still achieve the first best outcome even if $D^{\prime} s$ mixed strategy is observable. However, as easily checked, the exact counterpart of proposition 3 fails ${ }^{16}$. Now, we can show that if $\delta$ is sufficiently large, we can achieve the full efficiency asymptotically.

Theorem 4 Assume that D's mixed strategy is observable. The, given $\pi\left(\leq \pi^{*}\right), \forall \beta \in(0,1)$, there is a discount factor $\underline{\delta}(\beta, \pi)$ such that $\forall \delta>\underline{\delta}(\beta, \pi)$ the following strategies and beliefs constitute a communicative equilibrium;

(i) At each period $t, P$ plays the strategy $\sigma_{t}^{P}\left(h_{t-1}, w_{l}\right)=0, \sigma_{t}^{P}\left(h_{t-1}, w_{h}\right)=1, \forall h_{t-1} \in$ $H_{t-1}, \forall t$, and D's posterior belief is formed by $\pi_{t}=1$ if $m_{t}=H$ and otherwise, $\pi_{t}=0$, and $D$ plays the strategy $\sigma_{t}^{D}\left(h_{t-1}, m_{t}\right)=\beta$, if $m_{t}=H, \forall h_{t-1} \in H_{t-1}, \forall t, 0<\beta<1$ and $\sigma_{t}^{D}\left(h_{t-1}, m_{t}\right)=1$, if $m_{t}=L$, until $m_{t}$ turns out to be false or $D$ deviates from $(i)$.

(ii) If it is revealed that $m_{t} \neq \omega_{t}$ or $D$ deviates from $(i), \sigma_{t+1}^{P}\left(h_{t}, \omega_{t+1}\right)=1$ and $\sigma_{t+1}^{D}\left(h_{t}, m_{t+1}\right)=$ $1, \forall h_{t} \in H_{t}, \forall \omega_{t+1} \in \Omega$ are played thereafter.

Proof. See the appendix.

The equilibrium feature suggested by this theorem has in common with Green and Porter that in either model, all players involved know that there will be no cheating in equilibrium, but they cannot play the first best all the time. Some level of efficiency loss is indispensable to discourage the incentive to cheat.

Now, we will compare the result of this section with that of section 3. Full efficiency can be achieved when private information is fully revealed. However, suppose partial information only is revealed i.e., $\alpha^{*}>0$. As $\delta$ approaches 1 , the plaintiff is checked up less frequently, making a false announcement with the same frequency, so that $P$ gets better off, leaving $D^{\prime} s$ welfare unimproved. But as long as $\alpha^{*}>0$, the first best outcome cannot be approached 
even if $\delta \rightarrow 1$. This is easily seen by the observation that if $\alpha^{*}>0$, the players are absorbed in the permanent punishment regime within a finite period with probability 1 as long as $\beta^{*} \neq 0$, because $\operatorname{Prob}[$ The players enter into the punishment regime in a finite period.] $=$ $\sum_{t=1}^{\infty} \operatorname{Prob}[$ The players enter into the punishment regime in t period.] $=(1-\pi) \alpha \beta+\{1-$ $(1-\pi) \alpha \beta\}(1-\pi) \alpha \beta+\{1-(1-\pi) \alpha \beta\}^{2}(1-\pi) \alpha \beta+\cdots=1$. Therefore, for any $\delta<1$, efficiency loss incurred in the punishment regime is not negligible, so that full efficiency cannot be approximated if $\alpha^{*}>0$.

\section{Related literature}

Townsend (1982) showed that in the presence of private information, both agents in a repeated relationship could benefit from trading with each other, whereas they could not in a one-shot relationship. Even if the result of Townsend seems to have much in common with ours, it should be noted that it relies critically on the assumption of risk-aversion. In Townsend, it is through the principle of risk sharing that both agents can get away from status quo. So, if both agents are risk neutral as assumed in our model, the result of Townsend cannot be sustained. On the other hand, a unique feature that distinguishes our model from Townsend is the ability of the uninformed party to check up on the informed party. This ends up with a different result that any outcome other than the babbling equilibrium cannot be supported as an equilibrium in any finite period.

Sobel (1985) addressed the issue of reputation in a repeated cheap talk game. The crucial difference of his model from ours is that private information is initially fixed rather than drawn independently in each period. This difference in informational structure in a repeated game leads to different implications on how to build a reputation. In his model, the sender can build a reputation by mimicking the equilibrium behavior of a good type since there is no specific monitoring technology available to the receiver, while in our model, he can build his reputation by surviving receiver's test. So, once it falls, a reputation cannot be recoverable in Sobel's sense, while it can in our sense by passing the next test. Sobel showed that, in this context, it could pay to build up a reputation by consistently providing accurate information even in a finitely repeated relationship.

Nalebuff and Scharfstein (1987) considered tests that directly discover an agent's private information. They showed that even if the cost of testing made it usually impossible to achieve a first best allocation in models with asymmetric information, the full information equilibrium could be approximated in the limit by using a more expensive test less frequently 
with an increased penalty for failure. This asymptotic full efficiency result comes from the assumption that all the variables consisting of the test technology, including the probability of the test to be taken and an expenditure on the test are publicly observable as part of the contract, which is consistent with our result in section 4 .

\section{Conclusion}

In this paper, I have succeeded in showing the effectiveness of cheap talk in the infinitely repeated litigation game. Since the repeat players will care about not only the current payoff but also the future payoff, cheap talk can be effective in eliciting true information from the informed party.

The merit of this paper is that the outcome is consistent with the phenomena that we observe in the real world: First, it provides one possible answer for why there is little litigation between repeat players. Even though accidents within the firm occur quite often, the parties rarely go to trial, rather end up with agreement in compensation by the union being honest in reporting its damage amount and the firm believing it. Another plausible scenario would be that repeat players fear to terminate mutual benefit from their ongoing relationship that is expected to be continued. However, in some cases the outside option of terminating the relationship may be unavailable to the players; for example, dealing with the government cannot be discontinued. This model encompasses such a situation. Second, this paper can also explain the actual trial, if rare. Cheap talk is inherently so unbinding that reputation effect only is not enough to make it perfectly credible. Accordingly, sporadic checking of credibility by triggering a litigation would be necessary.

The basic intuition and principle of this model could be applied to a larger class of repeated games, even if the analysis hitherto has been made on a cheap talk game. For example, consider the Green and Porter model. The crucial weakness of that model is that in equilibrium, players should punish each other if they observe an unfavorable signal, even if they know that the other player did not deviate from the collusive behavior, to give an incentive to keep engaging in collusive behavior. However, if it is possible to monitor the other player's behavior with some costs, say through a market survey, we can get a more effective punishment scheme that inflicts a punishment only on the deviant.

Despite of some virtues of our punishment scheme that were mentioned above, I must admit that it is far from the most efficient one. So, it is not difficult to recognize that one of the promising challenges in the future research will be to construct the most efficient 
punishment scheme to support better outcomes as an equilibrium. On the other hand, imposing stationarity overly restricts the strategy space, which mitigates the power of cheap talk. So, it would be essential to allow for nonstationary strategies to achieve a higher degree of efficiency.

This model can possibly be modified and extended in various directions. We can think of a learning model where each player can learn the other player's strategy and some parameter values in the course of playing the litigation game repeatedly. We may introduce another kind of uncertainty into this model. As Galanter (1974) noted, repeat players must establish a reputation as "tough". A reputation as a "combatant" can serve as an asset to increase his bargaining power. If the defendant believes even with some small probability that the plaintiff is very "combatant" in the sense that he is not perfectly rational-minded, rather has a strong tendency of rejecting a low settlement offer, they will be more likely to coordinate, as in Kreps and Wilson (1982b). Also, we may think that even if the parties involved in a legal dispute themselves are one-shot players, the information one party possesses can be transmitted effectively by means of a repeat player, say, a lawyer. Therefore, we may consider a model with a long-run player, a lawyer, being interposed between two short-run players, a plaintiff and a defendant. However, in many cases, attorneys making personal injury claims are in a significant conflict of interest with their clients, on the contrary to the implicit assumption made in this paper that a lawyer is a mere representative of his client who acts only on behave of him. There are several sources where this conflict comes from. First, since the plaintiff is usually short-sighted, all the plaintiff cares about is his immediate gain. Therefore, he usually has an incentive to exaggerate the value of his damage inflicted, which may not be consistent with his attorney's incentive to maintain his good reputation. Even apart from reputational consideration, a quick settlement is often in the lawyer's interest, while waiting the defendant out is often in the client's interest. Therefore, it would be an interesting extension of this model if we consider a model of three-person pre-trial negotiation game among a plaintiff, his attorney and a defendant that incorporates the conflict of interest between the plaintiff and his attorney. This issue is extensively discussed in Kim (1992) but I will omit it here.

From the viewpoint of repeated game theory, this model is quite different with the Kreps-Wilson-Fudenberg-Levine type of repeated game with incomplete information (Kreps and Wilson, 1982b; Fudenberg and Maskin, 1986; Fudenberg and Levine, 1989). In their model, private information is initially fixed, so that once it is known to the uninformed party, the game they play becomes one of complete information. Then, even in the finitely repeated 
game, cooperation is possible by virtue of the incentive of the bad type to mimic the good type. But, if private information is successively realized at the beginning of each period, we can see that the best the uninformed can do at each period is to do the best in the stage game. So, the only equilibrium is the simple repetition of one-shot equilibrium. How can the players reach cooperation in this situation? Cheap talk fills the role!

\section{Appendix}

Proof of proposition 2

Clearly, in equilibrium, $\sigma_{t}^{P}\left(h_{t-1}, w_{h}\right)=1$ and $\sigma_{t}^{D}\left(h_{t-1}, L\right)=1$. By the definition of the stationary Markov strategy, the following pure strategies are well-defined; (i) $\tilde{\sigma}^{P} \equiv$ $\sigma_{t}^{P}: H_{t-1} \times \Omega \rightarrow \Delta(M) \ni \sigma_{t}^{P}\left(h_{t-1}, w_{h}\right)=1$ and $\sigma_{t}^{P}\left(h_{t-1}, w_{l}\right)=0, \forall h_{t-1} \in H_{t-1}, \forall t$ (ii) $\tilde{\tilde{\sigma}}^{P} \equiv \sigma_{t}^{P}: H_{t-1} \times \Omega \rightarrow \Delta(M) \ni \sigma_{t}^{P}\left(h_{t-1}, w_{h}\right)=1$ and $\sigma_{t}^{P}\left(h_{t-1}, w_{l}\right)=1, \forall h_{t-1} \in H_{t-1}, \forall t$ (iii) $\tilde{\sigma}^{D} \equiv \sigma_{t}^{D}: H_{t-1} \times M \rightarrow \Delta(S) \ni \sigma_{t}^{D}\left(h_{t-1}, H\right)=0$ and $\sigma_{t}^{D}\left(h_{t-1}, L\right)=1, \forall h_{t-1} \in H_{t-1}, \forall t$ (iv) $\tilde{\tilde{\sigma}}^{D} \equiv \sigma_{t}^{D}: H_{t-1} \times M \rightarrow \Delta(S) \ni \sigma_{t}^{D}\left(h_{t-1}, H\right)=1$ and $\sigma_{t}^{D}\left(h_{t-1}, L\right)=1, \forall h_{t-1} \in H_{t-1}, \forall t$

Suppose $\tilde{\sigma}^{D}$ is an equilibrium strategy for $D$. Then, $P^{\prime} s$ expected utility of choosing $\tilde{\tilde{\sigma}}^{P}$ is $U^{P}\left(\tilde{\tilde{\sigma}}^{P}, \tilde{\sigma}^{D}, \omega\right)=s_{h}>\pi s_{h}+(1-\pi) s_{l}=U^{P}\left(\tilde{\sigma}^{P}, \tilde{\sigma}^{D}, \omega\right)$. So, $\tilde{\tilde{\sigma}}^{P}$ is the unique best response to $\tilde{\sigma}^{D}$. This violates the definition of the communicative equilibrium. Q.E.D.

\section{Proof of proposition 3}

Suppose $\tilde{\sigma}^{P}$ is an equilibrium strategy for $P$. Then, $\tilde{\sigma}^{D}$ is the unique best response to $\tilde{\sigma}^{P}$ because $U^{D}\left(\tilde{\sigma}^{P}, \tilde{\sigma}^{D}, \omega\right)=-\left\{\pi s_{h}+(1-\pi) s_{l}\right\}>-\left\{\pi\left(x_{h}+c_{d}\right)+(1-\pi) s_{l}\right\}=U^{D}\left(\tilde{\sigma}^{P}, \tilde{\tilde{\sigma}}^{D}, \omega\right)$ by the assumption $[\mathrm{A}]$. This contradicts proposition 2. Q.E.D.

\section{Proof of proposition 4}

By the definition of the stationary Markov strategy, the following strategy and $D^{\prime} s$ posterior belief are well-defined; (i) $\sigma^{P}(\alpha) \equiv \sigma_{t}^{P}: H_{t-1} \times \Omega \rightarrow \Delta(M) \ni \sigma_{t}^{P}\left(h_{t-1}, w_{h}\right)=$ 1 and $\sigma_{t}^{P}\left(h_{t-1}, w_{l}\right)=\alpha, \forall h_{t-1} \in H_{t-1}, \forall t$ (ii) $\tilde{\pi}(\alpha) \equiv \pi_{t}: H_{t-1} \times M \rightarrow[0,1] \ni \pi_{t}\left(h_{t-1}, "\right.$ " $\left.L "\right)=$ 0 and $\pi_{t}\left(h_{t-1}, " H "\right)=\frac{\pi}{\hat{\pi}(\alpha)}, \forall h_{t-1} \in H_{t-1}, \forall t$

Suppose $W=W^{p}$. If $\tilde{\pi}\left(\alpha^{*}\right)<\pi^{*}, \beta^{*}=1$, because $U^{D}\left(\sigma^{P}\left(\alpha^{*}\right), \tilde{\tilde{\sigma}}^{D}, \omega\right)=-\tilde{\pi}(\alpha)\left(x_{h}+c_{d}\right)-$ $(1-\tilde{\pi}(\alpha)) s_{l}>-s_{h}=U^{D}\left(\sigma^{P}\left(\alpha^{*}\right), \tilde{\sigma}^{D}, \omega\right)$ from the definition of $\pi^{*}$. Similarly, if $\tilde{\pi}\left(\alpha^{*}\right)>\pi^{*}$, $\beta^{*}=0$. Therefore, $\tilde{\pi}\left(\alpha^{*}\right)=\pi^{*}$, i.e., $\alpha^{*}=\frac{\left(x_{h}+c_{d}-s_{l}\right) \pi}{\left(s_{h}-s_{l}\right)(1-\pi)}$, if $\beta^{*} \in(0,1)$. Conversely, if $\tilde{\pi}\left(\alpha^{*}\right)=\pi^{*}$, 
i.e., $\alpha^{*}=\frac{\left(x_{h}+c_{d}-s_{l}\right) \pi}{\left(s_{h}-s_{l}\right)(1-\pi)}, W=W^{p}=\pi\left(x_{h}+c_{d}-s_{l}\right)+s_{l}$ from the equations $(7)-(10),(12)$ and (14). This implies that $\alpha^{*}=\frac{\left(x_{h}+c_{d}-s_{l}\right) \pi}{\left(s_{h}-s_{l}\right)(1-\pi)}$ and $W=W^{p}=\pi\left(x_{h}+c_{d}-s_{l}\right)+s_{l}$ satisfy the system of equations. Also, it is trivial to show that there exists only one root for $\alpha \in(0,1)$. Q.E.D.

\section{Proof of theorem 2}

Form the equation (13), $V_{L}^{l}=V_{L}^{h}$. Suppose $\lim _{N \rightarrow \infty} \beta^{*}(N, \delta, \pi)=0$. Then, $\lim _{N \rightarrow \infty} V_{L}^{l}(N, \delta, \pi)=$ $(1-\delta) s_{l}+\delta \lim _{N \rightarrow \infty} V(N, \delta, \pi)$ and $\lim _{N \rightarrow \infty} V_{L}^{h}=(1-\delta) s_{h}+\delta \lim _{N \rightarrow \infty} V(N, \delta, \pi)$. As long as $\delta<1, \lim _{N \rightarrow \infty} V_{L}^{l}(N, \delta, \pi)<\lim _{N \rightarrow \infty} V_{L}^{h}(N, \delta, \pi)$, which implies that $\exists$ a large $M$ such that $\forall N>M, V_{L}^{l}(N, \delta, \pi)<V_{L}^{h}(N, \delta, \pi)$. Then, $\forall N>M, \alpha^{*}(N, \delta, \pi)=0$. This is a contradiction to proposition 3. Therefore, $\lim _{N \rightarrow \infty} \beta^{*}(N, \delta, \pi)=\underline{\beta}(\delta, \pi)>0$. Q.E.D.

\section{Proof of theorem 3}

(i) As $N \rightarrow \infty$, the equation (13) is reduced to

$$
\begin{aligned}
(1-\delta) s_{l}+\delta V= & \{1-\underline{\beta}(\delta, \pi)\}\left\{(1-\delta) s_{h}+\delta V\right\}+ \\
& \underline{\beta}(\delta, \pi)\left[(1-\delta) s_{l}+\delta\left\{\pi\left(x_{h}-c_{p}-s_{l}\right)+s_{l}\right\}\right] .
\end{aligned}
$$

Since $\lim _{\delta \rightarrow 1} V \neq \pi\left(x_{h}-c_{p}-s_{l}\right)+s_{l}, \lim _{\delta \rightarrow 1} \underline{\beta}(\delta, \pi)=0$.

(ii) Since $V_{L}^{l}=V_{L}^{h}, V_{L}=V_{L}^{l}=(1-\delta) s_{l}+\delta V$. So, the equation (1) is reduced to

$$
V=\pi\left\{s_{h}-\left(s_{h}-x_{h}+c_{p}\right) \beta\right\}+(1-\pi) s_{l} .
$$

From (i), $\lim _{\delta \rightarrow 1} V=\pi\left(s_{h}-s_{l}\right)+s_{l}$. Q.E.D.

\section{Proof of theorem 4}

Fix $\beta^{*}$ and $\pi$. Then, we only need to choose $\underline{\delta}\left(\beta^{*}, \pi\right)$. Suppose $\alpha=0$ in equilibrium. Then, the followings should hold;

$$
\begin{gathered}
V=\pi V_{H}+(1-\pi) V_{L} \\
V_{H}=\left(1-\beta^{*}\right)\left\{(1-\delta) s_{h}+\delta V\right\}+\beta^{*}\left\{(1-\delta)\left(x_{h}-c_{p}\right)+\delta V\right\} \\
V_{L}=V_{L}^{l} \\
V_{L}^{l}=(1-\delta) s_{l}+\delta V
\end{gathered}
$$




$$
\begin{gathered}
V^{p}=\pi\left(x_{h}-c_{p}\right)+(1-\pi) s_{l} \\
W=\pi W_{H}+(1-\pi) W_{L} \\
\left.W_{H}\right|_{\beta=\beta^{*}}=\left(1-\beta^{*}\right) W_{H}^{h}+\beta^{*} W_{H}^{l} \\
\left.W_{H}\right|_{\beta \neq \beta^{*}}=(1-\delta)\left\{(1-\beta) s_{h}+\beta\left(x_{h}+c_{d}\right)\right\}+\delta W^{p} \\
W_{L}=(1-\delta) s_{l}+\delta W \\
W_{H}^{h}=(1-\delta) s_{h}+\delta W \\
W_{H}^{l}=(1-\delta)\left(x_{h}+c_{d}\right)+\delta W \\
W^{p}=\pi\left(x_{h}+c_{d}\right)+(1-\pi) s_{l}
\end{gathered}
$$

$\underline{\delta}\left(\beta^{*}, \pi\right)$ should be chosen so that (i) $V_{L}^{l}>V_{L}^{h}$ (ii) $\left.\beta^{*} \in \arg \min W_{H}\right|_{\beta}$. After simple algebra, we obtain

$$
\begin{gathered}
V=\pi s_{h}+(1-\pi) s_{l}-\pi \beta^{*}\left(s_{h}-x_{h}+c_{p}\right) \\
W=\pi\left\{\left(1-\beta^{*}\right) s_{h}+\beta^{*}\left(x_{h}+c_{d}\right)\right\}+(1-\pi) s_{l}
\end{gathered}
$$

So, $V_{L}^{l}-V_{L}^{h}=\delta \beta^{*}\left(1-\beta^{*}\right) \pi\left(s_{h}-x_{h}+c_{p}\right)-(1-\delta)\left(1-\beta^{*}\right)\left(s_{h}-s_{l}\right)$. Therefore, $\exists \delta_{1}(\beta, \pi) \ni$ $\forall \delta>\delta_{1}(\beta, \pi), V_{L}^{l}-V_{L}^{h}>0$. Similarly, $\left.W_{H}\right|_{\beta=\beta^{*}}-\left.W_{H}\right|_{\beta \neq \beta^{*}}=(1-\delta)\left(\beta-\beta^{*}\right)\left(x_{h}+c_{d}-s_{h}\right)-$ $\delta \pi\left(1-\beta^{*}\right)\left(x_{h}+c_{d}-s_{h}\right)$. Therefore, $\exists \delta_{2}(\beta, \pi) \ni \forall \delta>\delta_{2}(\beta, \pi),\left.W_{H}\right|_{\beta=\beta^{*}}-\left.W_{H}\right|_{\beta \neq \beta^{*}}<0$. Taking $\underline{\delta}(\beta, \pi)=\max \left\{\delta_{1}(\beta, \pi), \delta_{2}(\beta, \pi)\right\}$ completes the proof. Q.E.D.

\section{References}

[1] Aesop. The Fables of Aesop. New York: Evert Duyckinck, 1813

[2] Crawford, V. and Sobel, J. "Strategic Information Transmission." Econometrica, Vol. 50 (1982), pp. 1431-1451

[3] Farrell, J. "Cheap Talk, Coordination, and Entry." RAND Journal of Economics, Vol.18 (1987), pp. 34-39

[4] Farrell, J. and Gibbons, R. "Cheap Talk can Matter in Bargaining." Journal of Economic Theory, Vol. 48 (1989), pp. 221-237

[5] Farrell, J. and Saloner, G. "Standardization, Compatibility, and Innovation." RAND Journal of Economics, Vol. 16 (1985), pp. 70-83 
[6] Fudenberg, D. and Levine, D. "Reputation and Equilibrium Selection in Games with Patient Player." Econometrica, Vol. 57 (1989), pp. 759-778

[7] Fudenberg, D. and Maskin, E. "The Folk Theorem in Repeated Games with Discounting and with Incomplete Information." Econometrica, Vol. 54 (1986), pp. 533-554

[8] Galanter, M. "Why the "Haves" come out ahead: Speculations on the Limits of Legal Change." Law and Society, Vol. 9 (1974), pp. 95-159

[9] Green, E. and Porter, R. "Non-cooperative Collusion Under Imperfect Price Information." Econometrica, Vol. 52 (1984), pp. 87-100

[10] Howard, R. Dynamic Programming and Markov Processes. New York: M.I.T. and John Wiley and Sons, 1960

[11] Kim, J. "Does Cheap Talk Matter in Pre-trial Negotiation?" Seoul Journal of Economics, Vol. 5 (1993), pp. 301-315

[12] —. "Three Essays in Pre-Trial Negotiation," Ph.D. dissertation, Department of Economics, Stanford University, 1992

[13] Kreps, D. and Wilson, R. "Sequential Equilibrium." Econometrica, Vol. 50 (1982a), pp. 863-894

[14] —. "Reputation and Imperfect Information." Journal of Economic Theory, Vol. 27 (1982b), pp. 253-279

[15] Matthews, S. "Veto Threats: Rhetoric in a Bargaining Game." Quarterly Journal of Economics, Vol. 104 (1989), pp. 348-369

[16] Matthews, S. and Postlewaite, A. "Pre-Play Communication in Two-Person Sealed-Bid Double Auctions." Journal of Economic Theory, Vol. 48 (1989), pp. 238-263

[17] Nalebuff, B. and Scharfstein, D. "Testing in Models of Asymmetric Information." Review of Economic Studies, Vol. 54 (1987), pp. 265-277

[18] Sobel, J. "A Theory of Credibility." Review of Economic Studies, Vol. 52 (1985), pp. $557-573$

[19] Townsend, R. "Optimal Multiperiod Contracts and the Gain from Enduring Relationships under Private Information." Journal of Political Economy, Vol. 90 (1982), pp. 1166-1186 
[20] Williams, G. Lawyer's Handbook for Effective Negotiation and Settlement. Washington, D.C.: The Legal Education Institute, 1981 


\section{Footnotes}

1. There have been some informal discussions on the importance of communication in pre-trial negotiation. For example, Williams (1981) asserted that the purpose of communicating information is to cause the other side to feel and believe that the negotiator has extraordinary high expectations in this case, that the other side will have to make substantial concessions if it is going to settle the case, etc..

2. Any claim which is costless, unbinding and nonverifiable can be referred to as " cheap talk".

3. Even if $90 \%$ of the United States labor force is covered by a system of compulsory compensation of injured workers without regard to fault, which is known as a workers' compensation system, some states still do not have a compulsory system and others have elective coverage. Also, in some states there are size-of-firm restrictions that exempt small firms from joining the system and in most states farm workers are excluded from coverage.

4. It is assumed that $P$ incurs no legal costs by filing an action. This assumption implies that $P$ will always bring an action because regardless of the $D^{\prime} s$ choice of strategy, $P$ will receive a higher return from filing an action.

5. Even if the message space is assumed to be unrestricted, there is not much that can be said. In fact, it is sufficient to consider the space of equilibrium messages $\{$ " $H$ ", " $L "\}$.

6. The magnitude of $x_{l}+c_{d}$ is not important in this analysis. When $D$ determines his settlement offer, he does not have to worry about being found in court by his offer being rejected because $P_{l}$ accepts both $s_{l}$ and $s_{h}$.

7. The sequential equilibrium is defined with respect to the assessment, a pair of a strategy and a system of beliefs, but here the specification of beliefs will be sometimes omitted as long as there is no chance of confusion.

8. If cheap talk does not affect the payoff-relevant action meant to be played in equilibrium, it will still be called an uncommunicative equilibiurm even if it conveys some meaningful message.

9. Single-period payoffs are received at the end of each period. 
10. The term "Markov strategy" here is a weaker concept than in the usual sense. By Markov strategy, I mean that, in a normal phase, the probability that $D$ plays $s_{l}$ at period $t$ depends only on the current message, while the probability that $\mathrm{P}$ announces " $\mathrm{H}$ " given $w_{l}$ depends only on the current information. Non-Markovian behavior in a punishment phase is not excluded by this definition.

11. It is guaranteed that $0<\alpha^{*}<1$ when $\pi \leq \pi^{*}$.

12. Sequential rationality requires that at each information set, the players must play optimally, given their beliefs, for the remainder of the game.

13. By one-sided efficiency, I mean that only one player gets the first best payoff in contrast to full efficiency, meaning that both players achieve the first best outcome.

14. For this analysis, we will restrict our attention to communicative equilibria.

15. This section was inspired by Jeroen Swinkels.

16. The reason is as follow; If $D^{\prime} s$ mixed strategy is observable, $P$ can induce $D^{\prime} s$ random play of $s_{l}$ in equilibrium even without lying with strictly positive probability, by punishing $D$ if he deviates from the agreed-upon mixed strategy $\beta$, which is impossible if $\beta$ is not observable. 